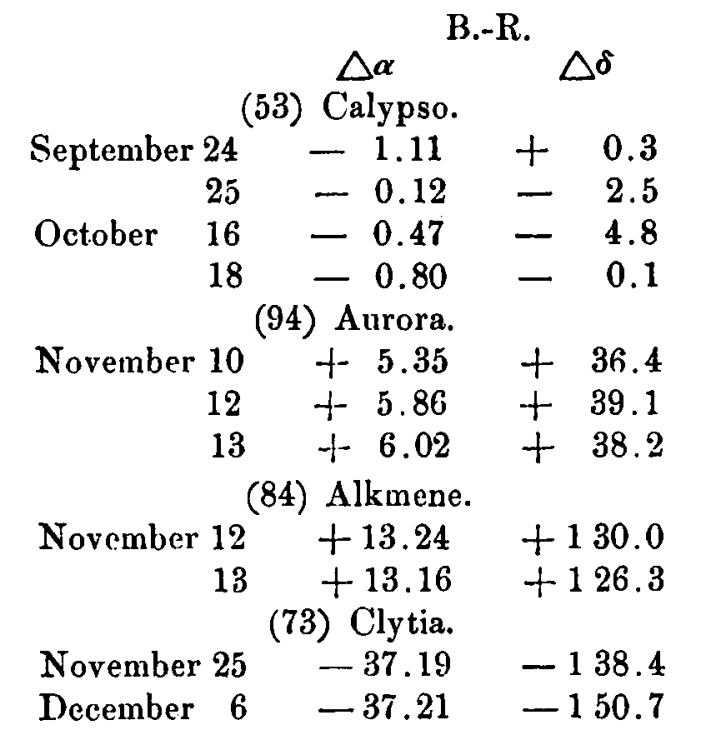

\title{
Schreiben des Herrn Prof. d'Arrest an den Herausgeber.
}

Den hellen Cometen von Coggia habe ich bisher am Filarmikrometer des Refraktors folgendermaassen beobachtet. Der Kern, gegenwärtig gleich * 8.5 Grösse, lässt sich, trotz des hier jetzt sehr hellen Nachthimmels, vorzüglich fixiren.

\begin{tabular}{|c|c|c|c|}
\hline $\begin{array}{l}1874 . \\
\text { Mai } 8\end{array}$ & $\begin{array}{l}\text { M. Zeil Kopenh. } \\
10^{\mathrm{h}} 23^{\mathrm{m}} 13^{\mathrm{s}}\end{array}$ & $\begin{array}{l}\text { A.R. Comet. } \\
6^{\text {h }} 22^{\mathrm{m}} 18^{\mathrm{s}} 39\end{array}$ & $\begin{array}{l}\text { Decl. Comel. } \\
+68^{\circ} 53^{\prime} 37^{\prime \prime} 7\end{array}$ \\
\hline 9 & $1017 \quad 8$ & 62244.48 & +685216.3 \\
\hline 12 & 101654 & 62424.35 & +68491.4 \\
\hline 13 & $1014 \quad 2$ & 6250.12 & +684814.3 \\
\hline 14 & 101417 & $\begin{array}{ll}625 & 41.77\end{array}$ & +684736.4 \\
\hline 15 & 101628 & 62625.36 & +68477.5 \\
\hline 18 & 103032 & 62855.55 & +684643.1 \\
\hline 19 & $1013 \quad 3$ & 62950.07 & +684645.7 \\
\hline 20 & 103543 & $\begin{array}{lll}630 & 48.89\end{array}$ & t- $6847 \quad 0.3$ \\
\hline 21 & 113451 & $\begin{array}{lll}631 & 54.08\end{array}$ & +684723.4 \\
\hline 22 & $1028 \quad 17$ & $632 \quad 53.72$ & t- 684752.3 \\
\hline 23 & 104214 & $634 \quad 0.34$ & +684829.2 \\
\hline
\end{tabular}

Der Vergleichungen sind in der Regel 6, wobei ich die Sterne so angenommen habe, nämlich von Mai 8 bis 15:

Mill. Ort 1874,0

* Argel. Ö $69726^{\mathrm{h}} 24^{\mathrm{m}} 59^{\mathrm{s}} 24+68049^{\mathrm{g}} 48^{\prime \prime} 3$

vom Mai 18 bis 23:

* Argel. Durchm. 447, Bonner Beob. Bd. VI, pag. 307, $6^{\mathrm{h}} 27^{\mathrm{m}} 55^{\mathrm{s}} 15+68^{n} 45^{\prime} 12^{\prime \prime} 3$.

Die ungewöhnlich grosse Schwierigkeit der Bahınbestimmung dieses Cometen besteht noch gegenwïrtig fort, während derselbe mit so geringer Geschwindigkeit seinen scheinbar verschlungenen Lauf fortsetzt. Aus den Positionen des ersten Monats hat neulich Cand. Svedstrup (aus Helsingör) die nachstehenden Elemente berechnet, welche nahezu in die Mitte fallen zwischen das zweite System von Holetschek und das von Dr. Dunír angegebene, nämlich:

$$
\begin{aligned}
& \text { T. .... } 1874 \text { Juli 11,1526 M. Zeit Greenwich. } \\
& \pi \ldots \ldots 271044^{\prime} 29^{\prime \prime} 4
\end{aligned}
$$

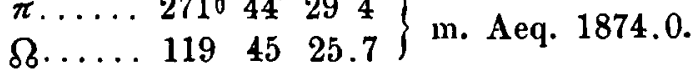

$$
\begin{aligned}
& \begin{array}{lllll}
i . \ldots . & 68 & 6 & 0.8 \text {. }
\end{array}
\end{aligned}
$$

$\log q \ldots 9,835913$.

$$
\text { Bewegung direkt. }
$$

Aus diesen Elcmenten finde ich für zwei meiner Positionen der letzten Nächte diese geringen Abweichungen:

$$
\begin{array}{cc}
\triangle \alpha \cos \delta & \triangle \delta \\
\text { Mai } 20-4^{3} 67 & -27^{\prime \prime} 4 \\
21-4.82 & -20.6
\end{array}
$$

während die publicirten Ephemeriden, in entgegengesetzten Richtungen, bereits grössere Abweichnngen von

\begin{tabular}{|c|c|c|c|c|}
\hline $\begin{array}{l}1874 . \\
\text { Mai } 8\end{array}$ & $\begin{array}{l}\text { M. Z Zeit. } \\
11^{1139^{\mathrm{m}} 7^{\mathrm{s}}}\end{array}$ & $\begin{array}{l}\text { AR. Comet. } \\
6^{\mathrm{h}} 22^{\mathrm{m}} 19^{\mathrm{s}} .29\end{array}$ & $\begin{array}{r}\text { Decl. Comel. } \\
+68^{0} 53^{\prime} 31^{\prime \prime} 5\end{array}$ & $\begin{array}{l}\text { Vergl } \\
3\end{array}$ \\
\hline 9 & 105232 & 62243.60 & +685218.3 & 5 \\
\hline 13 & $1034 \quad 1$ & $625 \quad 0.69$ & +684816.7 & \\
\hline 15 & 103923 & $\begin{array}{lll}62626.37\end{array}$ & +684712.0 & 7 \\
\hline
\end{tabular}
den Beobachtungen ergeben.

Ausser meinen obigen Beobachtungen sind, hier auf der Sternwarte, von Magister J. Dreyer noch die folgenden, gleichfalls mit dem Filarmikrometer des Refractors, angestellt worden: 
M. Zeit.

Al. Comel.

Deel. Comet. Vergl.

scheint, besonders im Anfang des Julimonats, so glän-

Mai $18 \quad 10^{\mathrm{b}} 45^{\mathrm{m}} 34^{\mathrm{s}} \quad 6^{\mathrm{b}} 28^{\mathrm{m}} 55^{\mathrm{s}} .87+68^{0} 46^{\prime} 40^{\prime \prime} 7 \quad 6$

$19 \quad 103241 \quad 62951.22+684647.6 \quad 6$

$\begin{array}{llllllllll}20 & 11 & 3 & 51 & 6 & 30 & 50.55 & +6847 & 0.8 & 5\end{array}$

$21 \quad 103949 \quad 63151.03+684723.85$

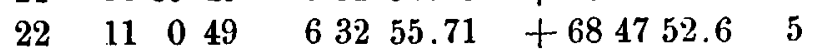

Dabei wurde der Comet mit denselben zwei Sternen, wie obenstehend, verglichen.

Dieser Comet, welcher in Juni, und, wie mir

zend werden soll, zeigte, während der Zeit obiger Beobachtungen, nur einförmiges Aussehen und langsamen Zuwachs der Helligkeit, doch lässt sich bereits ein etwa 100" langer Schweifansatz erkennen, welcher sich breit an den Cometenknopf anschliesst; Lichtausströmung aus dem Kerne haben wir jedoch bisher noch nicht bemerken können.

Kopenhagen, 1872 Mai 24.

d'Arrest.

\section{Preisaufgabe der K. Akademie der Wissenschaft in Copenbagen. \\ Question d'Astronomie.}

(Prix: la Médaille d'or de l'Académie.)

Il est, sous plusieurs rapports, important en Astronomie de connaitre les nombres qui ont servi de base aux anciennes recherches. Comme ils n'ont pas été rassemblès suivant un plan, mais qu'il faut, dans chaque cas donne, les chercher avec beaucoup de peine dans les grands ouvrages ou les traités spéciaux qui s'y rapportent, l'Académie désire de provoquer un travail où soient réunies, dans l'ordre chronologique de leur détermination, toutes les constantes dont on fait usage dans l'astronomie sphérique et théorique. Vu l'étendue de la matière, on se bornera à la période qui est comprise entre Ptolémée et la fin du XVIII siècle. Il ne sera pas nécessaire de soumettre à une critique Ia valeur intrinsèque des diverses constantes, mais il faudra les donner au complet et de manière qu'on puisse en $\mathrm{em}_{\overline{\text { }}}$ brasser l'ensemble. Seront en outre exclues les recherches spéciales concernant les monvements propres et les parallaxes des étoiles fixes, les satellites des planètes extérieures et les éléments des orbites des comètes.

Les réponses á cette question peuvent être écrites en latin, en français, en anglais, en allemand, en suédois et en danois. Les mémoires ne doivent pas porter le nom de l'anteur mais une devise, et être accompagnés d'un billet carcheté muni de la même devise, et renfermant le nom, la profession et l'adresse de l'auteur. Les membres de l'Académie qui demeurent en Danemark ne prennent point part au concours. La récompense accordée pour une réponse satisfaisante ì l'une des questions proposées, lorsqu'aucun autre prix n'est indiqué, est la médaille d'or de l'Académie, d'une valeur de 320 Conronnes.

Les mémoires doivent être adressés, avant la fin du mois d'Octobre 1875, an secrétaire de l'Acadénie, M. le Conseiller J. Japetns Sm. Steenstrup, professeur a l'Université de Copenhagne.

Découverte d'une nouvelle planète à l'Observatoire de Toulouse, par Mr. Perrotin. (Bulletin International, No. 246).

$\begin{array}{rccc} & \text { Temps moyen de Toulonse } & \text { Ascension droite } & \text { Déclinaison } \\ \text { Mai } 19 & \text { a } 10^{\mathrm{h}} 0^{\mathrm{m}} & 16 \mathrm{~h} 28^{\mathrm{m}} 30 \mathrm{~s} & -22^{\circ} 48^{\prime} \\ 20 & \text { à } 12.0 & 16.27 .28 & -22.47\end{array}$

Grandeur de la planète 11,5 .

\section{In halt.}

Zu No. 1995: C. Bruhns. Beobachtungen von Planeten und Cometen, angestellt auf der. Leipziger Slernwarte. 33. - d Arrest. Schreiben an den Herausgeber. 45. - Preisaufgabe der K. Akadenie der Wissenschaft in Copenhagen. 47. - Perrotin. Dicouverte d'une nouvelle planite à lobservatoire de Toulouse. 47.

Kiel 1874. Juni 10.

Druck von Fiencke \& Schachel in Kiel. 